\section{Fertilitätserhaltendes Management des Endometriumkarzinoms}

\begin{abstract}
Jüngere Frauen mit einem Endometriumkarzinom werden meist in einem frühen Tumorstadium diagnostiziert und eignen sich potenziell für eine fertilitätserhaltende Therapie. Erstmals untersuchten südkoreanische Mediziner den Langzeiteffekt einer oralen Behandlung dieser Patientinnen auf Progestin-Basis.
\end{abstract}

W ird bei Frauen unter 40 Jahren ein Endometriumkarzinom erstmals diagnostiziert, handelt es sich meist um gut differenzierte Adenokarzinome im Frühstadium ohne Beteiligung des Myometriums oder Lymphknoten-Metastasen. Angesichts der hohen Heilungschance in diesem Patientinnenkreis spielen Aspekte der Lebensqualität nach der Behandlung wie der Erhalt der Fertilität bei Frauen mit Kinderwunsch für die Therapieentscheidung eine wichtige Rolle.

$\mathrm{Zu}$ den gängigsten fertilitätserhaltenden Therapieoptionen gehört die orale Gabe von Progestinpräparaten wie $\mathrm{Me}$ thoxyprogesteronacetat (MPA) oder Megestrolacetat (MA). Bisher lagen jedoch keine wissenschaftlichen Publikationen zum langfristigen onkologischen Ergebnis vor. Die Forscher um Jeong-Yeol Park analysierten die Daten einer großen $\mathrm{Pa}$ tientinnenkohorte aus acht Krebszentren in Südkorea. 148 Patientinnen unter 40 Jahren genügten den Einschlusskriterien: endometroides Adenokarzinom des Endometriums, gut differenzierter Tumor (Grad 1), Stadium-I-Erkrankung gemäß FIGO, keine Beteiligung des Myometriums. Alle Patientinnen erhielten täglich oral MPA oder MA.

$77 \%$ erreichten ein komplettes Ansprechen (CR), davon $30 \%$ mit einem Rezidiv nach einem medianen Followup von 66 Monaten. Nach fünf Jahren betrug das rezidivfreie Überleben $68 \%$.
Für $23 \%$ der Patientinnen ohne kompletten Response verblieb nur die chirurgische Option. Als einzigen signifikanten Faktor im Zusammenhang mit dem ungenügenden Therapieansprechen machten die Autoren einen erhöhten Body-Mass-Index (über $25 \mathrm{~kg} / \mathrm{m}^{2}$ ) aus. Dieser erhöhte Wert korrelierte auch mit einem größeren Rezidivrisiko unter MPA im Vergleich zu MA. Eine Erhaltungstherapie und eine durchgemachte Schwangerschaft senkten das Rückfallrisiko hingegen signifikant.

Fazit: Das fertilitätserhaltende Management des Endometriumkarzinoms bei jüngeren Frauen auf der Basis von Progestin ist hocheffektiv und sicher. Ein Body-Mass-Index unter $25 \mathrm{~kg} / \mathrm{m}^{2}$ und die orale Gabe von MPA anstelle von MA, längere Therapiedauer und eine erfolgreiche Schwangerschaft erhöhten die Wahrscheinlichkeit für einen langfristigen Therapieerfolg.

Wolfgang Zimmermann

Park JY et al. Long-term oncologic outcomes after fertility-sparing management using oral progestin for young women with endometrial cancer (KGOG 2002). Eur J Cancer. 2013;49(4):868-74.

\title{
Endometriumkarzinom im Frühstadium: MikroRNA-Signaturen als Biomarker
}

\begin{abstract}
MikroRNA, d.h. nicht für Proteine kodierende, einsträngigen RNAs sind an der Regulation der Proteinexpression und damit vieler zellulärer Prozesse beteiligt und könnten anhand ihrer Expressionsmuster neue diagnostische Optionen beim endometrioiden Endometriumkarzinom eröffnen und sich als Prädiktor für die Prognose eignen.
\end{abstract}

D ie Pathogenese des endometrioiden Endometriumkarzinoms (EEC) als häufigster histologischer Form von Tumoren des Gebärmutterkorpus wurde in der Vergangenheit mit Mutationen in verschiedenen Genen wie PTEN, AKT, mTOR, KRAS und CTNNBI in Zusammenhang gebracht. Neue Einblicke in die Tumorpathogenese könnten Signaturen von MikroRNAs (miRNAs) eröffnen. Diese sind offenbar auf posttranskriptionaler Ebene an der Regulation der Genexpression beteiligt. MiRNAs lassen sich einfach auch aus dem Patien- tenblut gewinnen und auf ihre Expressionsmuster hin untersuchen.

Als Studienziel setzten sich Anna Torres und Kollegen die Definition von miRNA-Signaturen aus dem Tumorgewebe und Plasma und die Erforschung ihrer möglichen Eignung als diagnostische und prognostische Biomarker beim EEC. Sie gewannen Plasma- und Gewebeproben von 122 Frauen, davon 77 EEC-Fälle und 45 Kontrollpersonen. Sie erstellten die Expressionsprofile von 866 humanen und 89 humanen viralen miRNAs anhand von 24 Gewebsproben, gefolgt von einer quantitativen PCR-Validierung bei 104 Patientinnen. Es wurden auch die Expressionsmuster von 16 miRNAs aus 48 Plasmaproben untersucht.

Im Ergebnis gelang den Forschern anhand der identifizierten miRNA-Signaturen eine sichere Unterscheidung zwischen EEC- und gesundem Kontrollgewebe sowie den Plasmaproben. Unterschiede in der miRNA-Expression korrelierten auch signifikant mit dem Rezidivrisiko, dem Gesamtüberleben und progressionsfreien Krankheitsintervall.

Fazit: Die Expressionsmuster von $\mathrm{Mi}$ kroRNAs bergen aufgrund dieser Ergebnisse das Potenzial für nicht invasive Biomarker zur Detektion und Beurteilung der Prognose des endometrioiden Endometriumkarzinoms im Frühstadium.

Wolfgang Zimmermann

Torres A et al. Diagnostic and prognostic significance of miRNA signatures in tissues and plasma of endometrioid endometrial carcinoma patients. Int J Cancer. 2013;132(7):1633-45. 